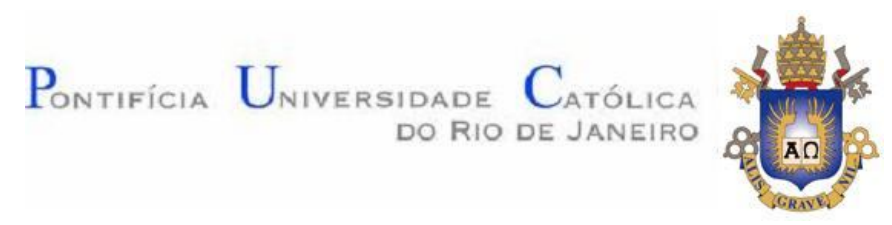

André Flaeschen

\title{
Arquitetura de Informação para Prestador de Serviços Logísticos especializado em Food Service
}

\begin{abstract}
Dissertação de Mestrado
Dissertação apresentada como requisito parcial para a obtenção do grau de Mestre pelo Programa de PósGraduação em Engenharia de Produção do Departamento de Engenharia Industrial da PUC - Rio.
\end{abstract}

Orientador: Prof. José Eugenio Leal Co-orientador: Prof. José Roberto de Souza Blaschek 


\title{
André Flaeschen
}

\section{Arquitetura de Informação para Prestador de Serviços Logísticos especializado em Food Service}

\section{Dissertação de Mestrado}

\begin{abstract}
Dissertação apresentada como requisito parcial à obtenção do título de Mestre (opção profissional) pelo Programa de Pós-Graduação em Engenharia de Produção do Departamento de Engenharia Industrial da PUC - Rio. Aprovada pela Comissão Examinadora abaixo assinada.
\end{abstract}

\author{
Prof. José Eugênio Leal \\ Orientador \\ Departamento de Engenharia Industrial - PUC-Rio \\ D.Sc. José Roberto de Souza Blaschek \\ Co-orientador \\ Departamento de Engenharia Industrial - PUC-Rio
}

D.Sc. Luiz Antônio de Moraes Pereira Banco Central do Brasil

D.Sc. Rodrigo Salvador Monteiro Universidade Federal do Estado do Rio de Janeiro - UFRJ

Dr. Ing. José Eugênio Leal Coordenador Setorial do Centro Técnico Científico - PUC-Rio 
Todos os direitos reservados. É proibida a reprodução total ou parcial do trabalho sem autorização da universidade, do autor e do orientador.

\section{André Flaeschen}

Graduou-se em Tecnologia em Processamento de Dados no Centro Universitário da Cidade e especializou-se em Tecnologia da Informação em Gestão de Negócios na Universidade do Estado do Rio de Janeiro (UERJ). Trabalha há onze anos com gerenciamento de projetos na área de Tecnologia da Informação na implantação de softwares de gestão corporativa. Atualmente exerce cargo de gerente de TI em empresa de Logística especializada em food service. Atua também como docente universitário em disciplinas relacionadas à Tecnologia da Informação, acumulando oito anos de experiência nas escolas de Administração e Marketing.

\section{Ficha catalográfica}

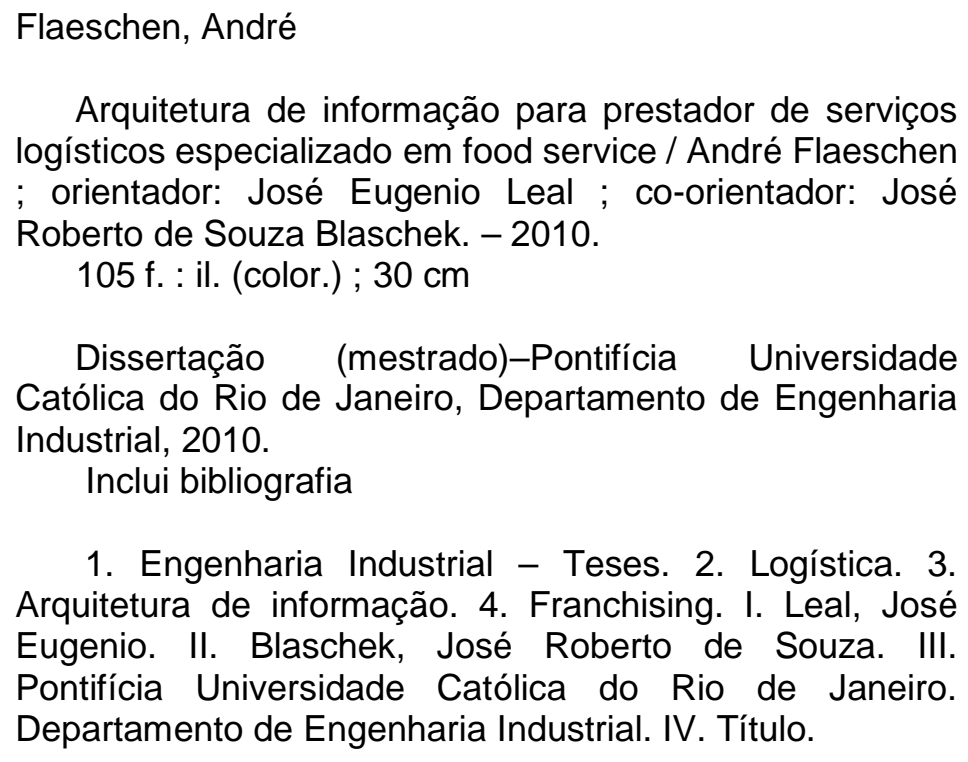

Arquitetura de informação para prestador de serviços logísticos especializado em food service / André Flaeschen ; orientador: José Eugenio Leal ; co-orientador: José Roberto de Souza Blaschek. - 2010.

105 f. : il. (color.) ; $30 \mathrm{~cm}$

Dissertação (mestrado)-Pontifícia Universidade Católica do Rio de Janeiro, Departamento de Engenharia Industrial, 2010.

Inclui bibliografia

1. Engenharia Industrial - Teses. 2. Logística. 3. Arquitetura de informação. 4. Franchising. I. Leal, José Eugenio. II. Blaschek, José Roberto de Souza. III. Pontifícia Universidade Católica do Rio de Janeiro. Departamento de Engenharia Industrial. IV. Título.

CDD: 658.5 
Para minha família, minha esposa Viviane e meus filhos Mariana e Gabriel. 


\section{Agradecimentos}

Ao Departamento de Engenharia Industrial da PUC-Rio e à Coordenação Central de Extensão, que possibilitou um aprendizado robusto e uma estadia prazerosa durante o Mestrado.

Aos professores do Mestrado Profissional em Logística da PUC-Rio pelo profissionalismo, conhecimento científico e incentivo depositados durante todo o curso.

Ao meu Co-orientador Prof. José Roberto Blaschek um agradecimento especial pela amizade, pela confiança em mim depositada e principalmente por dedicação e interesse na realização deste trabalho.

Ao meu Orientador e Professor José Eugênio Leal pela atenção dada e pela competência científica e profissional transmitida.

A todos os meus amigos e familiares que me estimularam ou me ajudaram de muitas formas.

A todos os colegas de mestrado que ingressaram juntos no ano de 2007, em particular ao Francisco Laranja e Alcione Dolavale.

A todas as pessoas que direta ou indiretamente contribuíram na minha busca pela capacitação e conclusão do curso. 


\section{Resumo}

Flaeschen, André; Leal, José Eugênio. Arquitetura de Informação para Prestador de Serviços Logísticos especializado em Food Service. Rio de Janeiro, 2010. 105p. Dissertação de Mestrado (Opção profissional) Departamento de Engenharia Industrial, Pontifícia Universidade Católica do Rio de Janeiro.

Nos últimos anos, o sistema de franchising, comum em redes de lanchonetes fast-food, vem ganhando maior destaque no cenário econômico mundial, incluindo o Brasil. Esse modelo de negócio, altamente padronizado, além de buscar expandir seu sistema geograficamente, precisa proteger seu maior ativo, a marca. Sua estruturação e gestão criam conflitos naturais de interesses e de assimetria de informações entre franqueador e franqueado, advindos da exploração coletiva de um mesmo ativo e da dispersão geográfica. Nesse sistema é muito importante ater-se às competências centrais. Como consequência, é usual a contratação de prestadores de serviços logísticos (PSL) para a coordenação e controle do fluxo de informações e mercadorias, percebidas como atividades de apoio ao negócio. Esta dissertação propõe uma arquitetura de informação capaz de apoiar os processos operacionais do PSL em estudo e monitorar seus fatores críticos de sucesso.

\section{Palavras-chave}

Logística; Arquitetura de Informação; Franchising 


\section{Abstract}

Flaeschen, André; Leal, José Eugênio (Advisor). Information Architecture for third party logistics specialized in Food Service. Rio de Janeiro, 2010. 105p. M. Sc. Dissertation - Departamento de Engenharia Industrial, Pontifícia Universidade Católica do Rio de Janeiro.

In the last years, the franchise system, common in fast food chains, is gaining greater prominence in the global economy scenery, including Brazil. This business model, highly standardized, in addition to seek to expand its system geographically, needs to protect its greatest asset, the brand. Its organization and management create natural conflicts of interests and asymmetric information between franchiser and franchisee, coming from the collective exploration of the same asset and geographic dispersion. In this system is very important to stick to the core competencies. As a result, it is usual to hire a third party logistics (3PL) providers to coordinate and control the flow of information and goods, perceived as activities to support the business. This thesis proposes an information architecture capable of supporting operational processes in the 3PL in study and monitor their critical success factors.

\section{Keywords}

Logistics; Information Architecture; Franchising 


\section{Sumário}

1. Introdução 13

1.1. Objetivos da Pesquisa 16

1.2. Delimitação do escopo da dissertação 16

1.3. A relevância do Tema 17

$\begin{array}{ll}\text { 1.4. Metodologia } & 18\end{array}$

$\begin{array}{ll}\text { 1.5. Estrutura da dissertação } & 18\end{array}$

2. Aspectos conceituais 20

2.1. O Sistema de franchising 20

2.1.1. O contrato no sistema de franchising 26

2.1.2. Integração dos membros da rede $\quad 27$

2.1.3. A evolução do sistema de franchising, segundo Mauro 29

2.1.3.1. 5 geração do franchising e os Fatores Críticos de Sucesso 30

2.1.4. Franchising no Brasil 30

2.1.5. Relacionamento entre franqueador e franqueado 32

2.1.5.1. Conflitos na relação franqueador/franqueado 33

2.2. Gestão da cadeia de suprimentos 35

2.2.1. Tecnologia da informação na gestão da cadeia de suprimentos 39

2.2.1.1. Mapa de TI da cadeia de suprimentos, segundo Chopra e Meindl 41

2.2.2. Gestão da cadeia de suprimentos em food-service 45

2.2.2.1. Terceirização de serviços logísticos 49

3. Revisão bibliográfica 52

3.1. Conceitos de Engenharia da Informação 52

3.2. A abordagem de James Martin 53

3.2.1. Pilares da engenharia da informação 55

3.3. Arquitetura Empresarial 55

3.3.1. Framework de Zachman 56

3.4. Planejamento da arquitetura empresarial 58

3.4.1. Fases do planejamento da arquitetura empresarial de Spewak 60

3.5. Tecnologias para Integração em tempo-real 62

3.5.1. Conceitos e componentes de Web Services 63 
3.5.2. Service-oriented Architecture (SOA) 65

3.6. Fatores Críticos de Sucesso (FCS) 67

3.6.1. Fontes primárias dos FCS 69

3.6.2. FCS para monitoramento de resultados $\quad 70$

4. Modelagem da Arquitetura de informação 71

4.1. Metodologia $\quad 71$

4.2. Iniciação do planejamento $\quad 72$

4.3. Modelo de negócio 73

4.3.1. A empresa em foco $\quad 73$

4.3.2. Áreas Funcionais $\quad 76$

4.3.3. Processos $\quad 78$

4.3.4. Matriz de processos por executivo 84

4.3.5. Fatores críticos de sucesso por área funcional 86

4.4. Arquitetura de dados 86

4.4.1. Agrupando as entidades em sistemas 90

4.5. Arquitetura de aplicação $\quad 95$

4.6. Arquitetura de tecnologia 96

5. Conclusões 99

$\begin{array}{ll}\text { Referências bibliográficas } & 101\end{array}$ 


\section{Lista de abreviaturas}

$\begin{array}{ll}\text { 3PL } & \text { - Third-party Logistics } \\ \text { ABF } & \text { - Associação Brasileira de Franchising } \\ \text { ABML } & \text { - Associação Brasileira de Movimentação e Logística } \\ \text { API } & \text { - Application Program Interfaces } \\ \text { APS } & \text { - Advanced Planning and Scheduling } \\ \text { CDR } & \text { - Centros de Distribuição Regional } \\ \text { COF } & \text { - Circular de Oferta de Franquia } \\ \text { COM } & \text { - Common Object Model } \\ \text { CORBA } & \text { - Common Object Request Broker Architecture } \\ \text { CRM } & \text { - Customer Relationship Management } \\ \text { CTRC } & \text { - Conhecimento de Transporte Rodoviário de Carga } \\ \text { DCOM } & \text { - Distributed COM } \\ \text { DRP } & \text { - Distribution Requirements Planning } \\ \text { EDI } & \text { - Eletronic Data Interchange } \\ \text { EI } & \text { - Engenharia de Informação } \\ \text { ERP } & \text { - Enterprise Resource Planning } \\ \text { FCS } & \text { - Fatores Críticos de Sucesso } \\ \text { HTTP } & \text { - Hypertext Transfer Protocol } \\ \text { IFA } & \text { - International Franchise Association } \\ \text { MES } & \text { - Manufactoring Execution System } \\ \text { MIT } & \text { - Massachusetts Institute of Technology } \\ \text { PSL } & \text { - Prestador de Serviço Logístico } \\ \text { RPC } & \text { - Remote Procedure Call } \\ \text { SAC } & \text { - Serviço de Atendimento ao Cliente } \\ \text { SC } & \text { - Supply Chain } \\ \text { SCM } & \text { - Supply Chain Management } \\ \text { SFA } & \text { - Sales Force Automation } \\ \text { SI } & \text { - Sistemas de Informação } \\ \text { SOA } & \text { - Service-oriented Architecture } \\ \text { SOAP } & \text { - Simple Object Access Protocol } \\ \text { TI } & \text { - Tecnologia da Informação } \\ \text { TMS } & \text { - Transportation Management System } \\ \text { XML } & \text { - Extensible Markup Language } \\ \text { W3C } & \text { - World Wide Web Consortiun } \\ \text { WMS } & \text { - Warehouse Management System } \\ \text { WS } & \text { - Web Services } \\ \text { WSDL } & \text { - Web Service Description Language } \\ & \end{array}$




\section{Lista de figuras}

Figura 1: Definição do Franchising 21

Figura 2: Franchising - Visão sistêmica 28

Figura 3: Cadeias de suprimentos interna, imediata e total 35

Figura 4: Escopo da gestão da cadeia de suprimentos 36

Figura 5: As três dimensões da gestão da cadeia de suprimentos $\quad 37$

Figura 6: Três eixos de atuação do SCM 38

Figura 7: Mapa de TI da cadeia de suprimentos 41

Figura 8: Mapa do sistema ERP da cadeia de suprimentos 43

Figura 9: Mapa de aplicativos analíticos da cadeia de suprimentos $\quad 45$

Figura 10: Distribuição física 48

Figura 11: Cadeia de Suprimentos da rede de Fast-Food 51

Figura 12: Engenharia da informação $\quad 54$

Figura 13: Componentes do planejamento da arquitetura empresarial 61

Figura 14: Mapa ampliado do planejamento de Spewak para o framework de Zachman 62

Figura 15: Organograma funcional do PSL em estudo 77

Figura 16: Fluxograma referente ao pedido de saída de produtos $\quad 79$

Figura 17: Fluxograma referente ao atendimento ao cliente 79

Figura 18: Fluxograma referente à compra de produtos 80

Figura 19: Fluxograma referente ao inventário de produtos $\quad 80$

Figura 20: Fluxograma referente à gestão financeira 81

Figura 21: Fluxograma referente ao controle fiscal 81

Figura 22: Fluxograma referente ao controle de viagem 82

Figura 23: Fluxograma referente ao retorno de viagem 82

Figura 24: Fluxograma referente à requisição de movimentação

de produto

82 


\section{Lista de Tabelas}

Tabela 1: Principais vantagens do Franchising 22

Tabela 2: Estatísticas do Franchising no Brasil 32

Tabela 3: Framework de Zachman $\quad 57$

Tabela 4: Áreas funcionais e processos 83

Tabela 5: Matriz de processos por executivos 85

Tabela 6: Entrada, processamento e saída, parte I 88

Tabela 7: Entrada, processamento e saída, parte II 89

Tabela 8: Relacionamento processos versus entidades, primeira iteração

Tabela 9: Relacionamento processos versus entidades, segunda iteração 92

Tabela 10: Relacionamento processos versus entidades, fluxo de dados I

Tabela 11: Relacionamento processos versus entidades, fluxo de dados II

Tabela 12: Relacionamento processos versus entidades, fluxo entre sistemas

Tabela 13: Arquitetura de tecnologia - distribuição de aplicativos e dados 